\title{
Dirac's Footsteps and Supersymmetry
}

\author{
P. Ramond \\ Institute for Fundamental Theory, Physics Department, \\ University of Florida, Gainesville, FL 32611, USA
}

I am not interested in proofs. I am only interested on how Nature works

P.A.M. Dirac

\begin{abstract}
One hundred years after its creator's birth, the Dirac equation stands as the cornerstone of XXth Century physics. But it is much more, as it carries the seeds of supersymmetry. Dirac also invented the light-cone, or "front form" dynamics, which plays a crucial role in string theory and in elucidating the finiteness of $N=4$ Yang-Mills theory. The light-cone structure of eleven-dimensional supergravity ( $N=8$ supergravity in four dimensions) suggests a group-theoretical interpretation of its divergences. We speculate they could be compensated by an infinite number of triplets of massless higher spin fields, each obeying a Dirac-like equation associated with the coset $F_{4} / S O(9)$. The divergences are proportional to the trace over a non-compact structure containing the compact form of $F_{4}$. Its nature is still unknown, but it could show the way to $M$-theory.
\end{abstract}

\section{Dirac's equation}

Much of modern physics starts with the Dirac equation. Its deceptively simple form

$$
\gamma^{\alpha} p_{\alpha} \Psi=\not p \Psi=0,
$$

obscured the significance of the simple algebraic relation for its anticommutator

$$
\{\not p, \not p\}=p^{2} .
$$

Today we understand this as the seed of supersymmetry, which could be called protosupersymmetry. In the context of string models, this relation turned out to be very potent. There, the momentum was generalized to a momentum density along the string

$$
P^{\alpha}(\sigma)=p^{\alpha}+\text { oscillators },
$$

suggesting that the momentum operator is the average of the momentum density

$$
p^{\alpha}=<P^{\alpha}>.
$$


If we assume that the Dirac matrices are themselves like dynamical variables (as envisioned by the Master himself[1])

$$
\Gamma^{\alpha}(\sigma)=\gamma^{\alpha}+\text { oscillators },
$$

with

$$
\gamma^{\alpha}=<\Gamma^{\alpha}>
$$

it is not unnatural[2] to expect that

$$
\gamma^{\alpha} p_{\alpha}=<\Gamma^{\alpha}><P_{\alpha}>\rightarrow<\Gamma^{\alpha} P_{\alpha}>,
$$

which generalizes the Dirac equation to the string. If we write

$$
\Gamma^{\alpha}(\sigma) P_{\alpha}(\sigma)=\sum_{n} F_{n} e^{i n \sigma}
$$

we find the superVirasoro algebra

$$
\begin{aligned}
\left\{F_{n}, F_{m}\right\} & =2 L_{n+m}, \\
{\left[L_{n}, F_{m}\right] } & =(2 n-m) F_{n+m}, \\
{\left[L_{n}, L_{m}\right] } & =(n-m) L_{n+m} .
\end{aligned}
$$

We should have read another of Dirac's papers 3 on the importance of $c$-numbers, which were found later by the late Joe Weiss. The rest is history as this algebra opened the Pandora box of theories with bosons and fermions, which we call supersymmetric.

\section{Divergences and Group Theory}

It is well-known how the running of the couplings in local field theory is related to the divergent part of some diagrams. For example, the one-loop beta function is given by

$$
\beta=\frac{11}{3} I_{a d j}^{(2)}-\frac{2}{3} I_{f}^{(2)}-\frac{1}{3} I_{H}^{(2)},
$$

where the $I_{a d j, f, H}^{(2)}$ are the quadratic Dynkin indices associated with the adjoint (for the gauge bosons), with spin one-half Weyl fermions, and with complex spin zero fields, respectively. These "external" group theoretical factors are given by

$$
\operatorname{Tr}\left(T_{r}^{A} T_{r}^{B}\right)=I_{r}^{(2)} \delta^{A B},
$$

wher $A, B$ run over the gauge group, and $T_{r}^{A}$ are the representation matrices in the $r$ representation. The other numerical factors stem from group theory of the "internal" space. As shown by Hughes [4], they can be understood for massless particles as, 


$$
\frac{1}{3}\left(1-12 h^{2}\right)
$$

where $h$ is the helicity of the particle circulating around the loop. In some sense the square of the helicity can be viewed as the quadratic Dynkin index of the light-cone [5] spin group, although in four dimensions, it is only $S O(2)$, which is not much of a group.

Curtright [ 6] generalized this notion when he considered loop integrals coming from theories in higher dimensions, where the spin light-cone little group is more substantial. For instance, the one loop vacuum polarization in $N=4$ Yang-Mills can be obtained directly in ten dimensions where the little group is $S O(8)$. Evaluate the loop integrals in four dimensions. The divergent part is proportional to

$$
(-1)^{f}\left(\frac{1}{3} I^{(0)}\left(\delta_{\mu \nu}^{(4)} q^{2}-q_{\mu} q_{\nu}\right)-\frac{4}{r} I^{(2)}\left(\delta_{\mu \nu}^{(D)} q^{2}-q_{\mu} q_{\nu}\right)\right),
$$

where $I^{(0)}$ is the dimension of the transverse little group representation, $r$ its rank, and $I^{(2)}$ is the quadratic Dynkin index of the same. In the case of $N=4$ Yang Mills, we have $D=10$ and the transverse little group is $S O(8)$. With its triality property, the group theoretical factors are the same for bosons and fermions. This is the genesis of the cancellation of ultraviolet divergences for that theory. It is the peculiar properties of the transverse little group that leads to ultraviolet finiteness [7, together with supersymmetry in the form of equality of fermions and bosons. This is true for all higher order Dynkin indices as well: since $S O(8)$ has rank four, it has three more independent Dynkin indices, of order 4,6 , and 8 , which are the same for bosons and fermions by triality. No other group has that property.

This is puzzling since all string theories stem from eleven-dimensional $M$ theory. The little group there is $S O(9)$, a totally unremarkable group, or so it seems. In eleven dimensions, supergravity is described by three fields $[\underline{8}$, the graviton $h_{\mu \nu}$, a Rarita-Schwinger fermion $\psi_{\mu}$ and a three-form boson $A_{\mu \nu \rho}$. Their physical degrees of freedom fall in three $S O(9)$ representations whose group-theoretical properties are summarized in the following table:

\begin{tabular}{|c|c|c|c|}
\hline $\begin{array}{c}\text { Field } \\
\text { irrep }\end{array}$ & $\begin{array}{c}\Psi_{i} \\
(1001)\end{array}$ & $\begin{array}{c}h_{i j} \\
(2000)\end{array}$ & $\begin{array}{c}A_{i j k} \\
(0010)\end{array}$ \\
\hline \hline$I^{(0)}$ & 128 & 44 & 84 \\
\hline$I^{(2)}$ & 256 & 88 & 168 \\
\hline$I^{(4)}$ & 640 & 232 & 408 \\
\hline$I^{(6)}$ & 1792 & 712 & 1080 \\
\hline$I^{(8)}$ & 5248 & 2440 & 3000 \\
\hline
\end{tabular}

The Dynkin indices have the remarkable property that they cancel between fermions and bosons 


$$
I_{\mathbf{1 2 8}}^{(k)}-I_{\mathbf{4 4}}^{(k)}-I_{\mathbf{8 4}}^{(k)}=0, \quad k=0,2,4,6,
$$

except for the highest invariant

$$
I_{\mathbf{1 2 8}}^{(8)}-I_{\mathbf{4 4}}^{(8)}-I_{\mathbf{8 4}}^{(8)}=-192 .
$$

This led Curtright to speculate that the theory is divergent because of this inequality. The lowest order divergent diagram that contains the eighth-order invariant is a three-loop four-graviton amplitude. While it is hopeless to calculate such a beast, this is the diagram for which there appears to be no local counterterm [9].

\section{$3 \quad$ Euler Triplets}

Sometime ago, it was found 10] that this pattern of group-theoretical partial cancellations among three representations generalized to other $S O(9)$ representations.

There are three equivalent embeddings of $S O(9)$ inside the exceptional group $F_{4}$, much like the I- U- and V-spins for the embedding of $S U(2) \times U(1)$ inside $S U(3)$. As a result, one can associate with each $F_{4}$ representation three $S O(9)$ representations, whose properties are summarized in the character formula [1]

$$
V_{\lambda} \otimes S^{+}-V_{\lambda} \otimes S^{-}=\sum_{c} \operatorname{sgn}(c) U_{c \bullet \lambda} .
$$

On the left-hand side, $V_{\lambda}$ is a representation of $F_{4}$ written in terms of its $S O(9)$ subgroup, $S^{ \pm}$are the two spinor representations of $S O(16)$ written in terms of its anomalously embedded subgroup $S O(9)$, and $\otimes$ denotes the normal Kronecker product of representations. On the right-hand side, the sum is over $c$, the three elements of the Weyl group which map the Weyl chamber of $F_{4}$ into the (three times larger) chamber of $S O(9)$. Finally $U_{c \bullet \lambda}$ denotes the $S O(9)$ representation with highest Dynkin weight $c \bullet \lambda$, where

$$
c \bullet \lambda=c\left(\lambda+\rho_{F_{4}}\right)-\rho_{S O(9)},
$$

and $\rho$ 's are the sum of the fundamental weights for each group, and $\operatorname{sgn}(c)$ is the index of $c$. Thus to each $F_{4}$ representation corresponds a triplet, called Euler triplet. The three representations of supergravity appear in the trivial case associated with the singlet of $F_{4}$. Since

$$
S O(16) \supset S O(9), \quad S^{+} \sim \mathbf{1 2 8}=\mathbf{1 2 8}, \quad S^{-} \sim \mathbf{1 2 8}^{\prime}=\mathbf{4 4}+\mathbf{8 4},
$$

the character formula reduces to

$$
128-44-84=128-44-84
$$


In general, the representations describe (in light-cone variables) fields with spin greater than two. For each $F_{4}$ representation with Dynkin labels $\left[a_{1} a_{2} a_{3} a_{4}\right]$ one obtains three $S O(9)$ representations listed in order of increasing dimensions:

$$
\left(2+a_{2}+a_{3}+a_{4}, a_{1}, a_{2}, a_{3}\right),\left(a_{2}, a_{1}, 1+a_{2}+a_{3}, a_{4}\right),\left(1+a_{2}+a_{3}, a_{1}, a_{2}, 1+a_{3}+a_{4}\right)
$$

For spinor representations, the fourth entry is an odd integer. Euler triplets for which the largest representation is the spinor have equal number of fermions and bosons; this occurs whenever both $a_{3}$ and $a_{4}$ are even integers or zero.

\section{Kostant Equation}

We find here again the long hand of Dirac, for the minus sign in the character formula suggests that it is the index formula for a Dirac-like operator. This is Kostant's operator 12 associated with the coset $F_{4} / S O(9)$. The Clifford algebra over this coset

$$
\left\{\Gamma^{a}, \Gamma^{b}\right\}=2 \delta^{a b}, a, b=1,2, \ldots, 16,
$$

is generated by $(256 \times 256)$ matrices, and the Kostant equation is defined as

$$
\not X \Psi=\sum_{a=1}^{16} \Gamma^{a} T^{a} \Psi=0,
$$

where $T_{a}$ are the $F_{4}$ generators not in $S O(9)$, with commutation relations

$$
\left[T^{a}, T^{b}\right]=i f^{a b i j} T^{i j} .
$$

These are conveniently expressed in terms of copies of 26 oscillators with the usual Bose-like commutation relations [13: $A_{0}^{[s]}, A_{i}^{[s]}, i=1, \cdots, 9, B_{a}^{[s]}, a=$ $1, \cdots, 16$, and their hermitian conjugates, and where $s=1,2,3$. Under $S O(9)$, the $A_{i}^{[s]}$ transform as $\mathbf{9}, B_{a}^{[s]}$ transform as $\mathbf{1 6}$, and $A_{0}^{[s]}$ is a scalar. Note that

the $B_{a}^{[s]}$ satisfy Bose-like commutation relations, even though they are $S O(9)$ spinors. The $F_{4}$ generators are then

$$
\begin{aligned}
T_{i j} & =-i \sum_{s=1}^{4}\left\{\left(A_{i}^{[s] \dagger} A_{j}^{[s]}-A_{j}^{[s] \dagger]} A_{i}^{[s]}\right)+\frac{1}{2} B^{[s] \dagger} \gamma_{i j} B^{[s]}\right\} \\
T_{a} & =-\frac{i}{2} \sum_{s=1}^{4}\left\{\left(\gamma_{i}\right)^{a b}\left(A_{i}^{[s] \dagger} B_{b}^{[s]}-B_{b}^{[s] \dagger} A_{i}^{[s]}\right)-\sqrt{3}\left(B_{a}^{[s] \dagger} A_{0}^{[s]}-A_{0}^{[s] \dagger} B_{a}^{[s]}\right)\right\} .
\end{aligned}
$$

One can just as easily have used the coordinate representation of the oscillators by introducing real coordinates $u_{i}$ which transform as transverse space vectors, $u_{0}$ as scalars, and $\zeta_{a}$ as the space spinors. It is amusing to note that the internal 
cordinates span three exceptional Jordan algebras, which have been the subject of much interest as possible charge spaces.

The solutions of Kostant's equation are then simply described by a chiral superfield 14. Listing only its highest weight components, it is of the form

$\Phi_{\vec{a}}=\theta^{1} \theta^{8} h\left(y^{-}, \vec{x}, u_{i}, \zeta_{a}\right)+\theta^{1} \theta^{4} \theta^{8} \psi\left(y^{-}, \vec{x}, u_{i}, \zeta_{a}\right)+\theta^{1} \theta^{4} \theta^{5} \theta^{8} A\left(y^{-}, \vec{x}, u_{i}, \zeta_{a}\right)$.

The three fields are polynomials in three sets of "internal" bosonic coordinates. A possible violation of the spin-statistics connection is avoided when the fields are even functions of the $\zeta_{a}$. This happens whenever $a_{3}$ and $a_{4}$ are even, but this is the case where each Euler triplet contains as many fermions as bosons. Hence there is an intriguing relation in this solution space between spin-statistics and equality between bosons and fermions. Supergravity is the trivial solution for which the three fields $h, A$, and $\psi$ are independent of the internal coordinates.

\section{A Zero Sum Game}

Since the Dynkin indices of the product of two representations satisfy the composition law

$$
I^{(n)}[\lambda \otimes \mu]=d_{\lambda} I^{(n)}[\mu]+d_{\mu} I^{(n)}[\lambda],
$$

where $d$ is the dimension, it follows that the deficit in $I^{(8)}$ is always proportional to

$$
d_{\lambda}\left(I_{S^{+}}^{(8)}-I_{S^{-}}^{(8)}\right)
$$

where $d_{\lambda}$ is the dimension of the $F_{4}$ representation that generates it. It is always of the same sign, so cancellation, if it happens will come only after summing over an infinite number of triplets.

Each triplet contains particles with spin greater than two. As we have shown [14, they cannot have mass since they do not assemble in massive little group multiplets. The only evasion route from the well documented difficulties [15] with higher spin massless fields interacting with gravity theories is to allow for an infinite number, but to this date no such theory has been put forth, perhaps with good reason.

If indeed the divergences of supergravity all stem from the deficit in the eighth order Dynkin, we are led to the bizarre equation

$$
\Delta I^{(8)}=-192 \sum_{a_{1}, a_{2}, a_{3}, a_{4}} d^{F_{4}}\left(a_{1}, a_{2}, a_{3}, a_{4}\right)=0,
$$

where $d^{F_{4}}\left(a_{1}, a_{2}, a_{3}, a_{4}\right)$ is the dimension of the $F_{4}$ representation with Dynkin labels $\left[a_{1} a_{2} a_{3} a_{4}\right]$. It can only vanish if the sum is over an infinite number of representations. In order to make sense of this at least two obstacles have to be 
surmounted. One is to specify the regularization procedure, and the second is to determine the subset of $F_{4}$ representations over which to take the sum.

We seek an algebraic structure which contains an infinite number of $F_{4}$ representations such that the (regulated) trace, or character, over the dimensions of these representations is zero. It would be wonderful if the triality properties of affine $E_{6}^{(1)}$ somehow became important. In quantum groups, zero traces can occur when the $q$ parameter is a root of unity ${ }^{1}$, so we are perhaps dealing with such an object. At any rate, it will be a miracle if such a structure exists, but then we are looking for a unique theory where miracles are expected on a daily basis.

Dimensions of $F_{4}$ representations are expressed as 24th order polynomials in their Dynkin indices. A zero-sum game can be clearly set up: assuming $\zeta$ function regularization, or a quantum group, find a subset of $F_{4}$ representations with zero total dimension. The existence of such a set would be strong indication for a finite structure underlying eleven-dimensional supergravity.

\section{Acknowledgments}

I wish to thank Professor H. Baer for his kind hospitality and giving this mere mortal the honor to speak at the commemoration of one of the Gods of Physics. This work was supported in part by the US Department of Energy under grant DE-FG02-97ER41029

\section{References}

[1] P. A. M. Dirac, Proc. Royal Soc. (London) A117 610: 1928

[2] P. Ramond, Phys. Rev. D3 2415: 1971

[3] P.A.M. Dirac, Rev. Mod. Phys. 34 592: 1962

[4] Richard J. Hughes, Phys.Lett. B97:246,1980

[5] P.A.M. Dirac, Rev. Mod. Phys.21 392:1948

[6] T. Curtright, Phys. Rev. Lett. 48, 1704(1982)

[7] Lars Brink, Olof Lindgren, Bengt E.W. Nilsson. Phys.Lett. B123, 323(1983); Stanley Mandelstam, Nucl.Phys. B213149(1983)

[8] E. Cremmer, B. Julia, J. Scherk, Phys. Lett B76, 409(1978)

[9] S. Deser and J. H. Kay, Phys. Lett. 76B, 400(1978); P. van Nieuwhenhuizen, Phys. Rep. 68, 189(1981)

[10] T. Pengpan and P. Ramond, Phys. Rep. 315. 137(1999)

\footnotetext{
${ }^{1}$ I thank E. Mukhin and P. di Francesco for an instructive discussion
} 
[11] B. Gross, B. Kostant, P. Ramond, and S. Sternberg, Proc. Natl. Acad. Scien., 8441 (1998)

[12] B. Kostant, Duke J. of Mathematics 100, 447(1999)

[13] T. Fulton, J. Phys. A:Math. Gen. 18, 2863(1985)

[14] Lars Brink, Pierre Ramond, Xiao-zhen Xiong, JHEP 0210:058,2002; hep-th 0207253

[15] M. A. Vasiliev, hep-th/0104246 and references therein 